\title{
Incidence of acute spinal cord injury in South Korea does not reflect a sizable number of traumatic spinal cord injuries
}

\author{
Ja-Ho Leigh $\mathbb{1}^{1,2} \cdot$ Han-Kyoul Kim $\mathbb{1}^{1,3} \cdot$ Moon Suk Bang $\mathbb{B}^{1,3}$
}

Received: 27 March 2020 / Revised: 8 April 2020 / Accepted: 14 April 2020 / Published online: 30 April 2020

(c) International Spinal Cord Society 2020

\section{To the Editor:}

We reviewed with great interest the article by Choi and colleagues regarding the incidence of acute spinal cord injury (SCI) and the associated complications of methylprednisolone use in a national population-based study in South Korea [1]. We consider this study to be valuable as it reported a 10-year incidence of acute SCI based on the data claimed in the national health insurance system. However, we would like to discuss several potential errors in the interpretation of the results, including the incidence and peak age of acute SCI in South Korea.

First, a sizable number of traumatic SCIs were excluded from the study sample. In South Korea, along with national health insurance, national workers' compensation and automobile insurance have the status as the nation's social security insurance and quasi-national insurance. National workers' compensation also provides coverage to $\sim 98 \%$ of the workers working in South Korea, similar to national health insurance, and automobile insurance is compulsory for all automobiles in Korea. As 2.29 million automobiles are in South Korea, there is currently one vehicle per 2.3 citizens $[2,3]$.

No differences exist for the medical procedures provide to patients using these different forms of insurance, including the administration of methylprednisolone in the emergency room after trauma. After receiving treatment such as for acute SCI, if the injury is identified as being subject to coverage from

Moon Suk Bang

msbang@snu.ac.kr

1 Department of Rehabilitation Medicine, Seoul National University College of Medicine, Seoul National University Hospital, Seoul, Republic of Korea

2 Department of Rehabilitation Medicine, Workers' Compensation and Welfare Incheon Hospital, Incheon, Republic of Korea

3 Department of Rehabilitation Medicine, National Traffic Injury Rehabilitation Hospital, Yangpyeong, Republic of Korea national workers' compensation or automobile insurance, national health insurance would not be charged, meaning that the injury would be excluded from the database of national health insurance - the database by Choi et al. [1].

The overall Incidence of SCIs that are covered by automobile insurance or national workers' compensation in South Korea has, to date, not been officially reported. However, when we compare a 2017 white paper examining automobile insurance claims for traumatic vertebral fractures with studies using national health insurance data involving the same target group, 30,586 automobile insurance and 123,820 national health insurance cases were reported, respectively $[4,5]$. Therefore, SCIs related to a damaged vehicle that are covered by automobile insurance represent a sizable number of cases. In addition, unpublished data from the industrial accident insurance reported that 289 patients with acute SCI who ended the treatment with the nine diagnosed diseases in 2017, and we expect that the annual incidence would be equivalent to this number.

Second, the peak incidence occurring among adults in their $50 \mathrm{~s}$ appears to be inaccurate. Existing data cannot rule out that this peak incidence is due to the exclusion of SCIs resulting from occupational and driving-related trauma in younger individuals.

Third, South Korea is a member country of the Organization for Economic Co-operation and Development. Thus, it has a system appropriate for developed countries to use for the prevention, emergency services, surgeries, and rehabilitation of SCI, which merits consideration when discussing international comparisons.

While national trauma data for medical standards is currently unavailable, we believe that Choi and colleagues' study represents an essential starting point when preparing data on SCI and neurotrauma.

\section{Compliance with ethical standards}

Conflict of interest The authors declare that they have no conflict of interest. 
Publisher's note Springer Nature remains neutral with regard to jurisdictional claims in published maps and institutional affiliations.

\section{References}

1. Choi SH, Sung C-h, Heo DR, Jeong S-Y, Kang C-N. Incidence of acute spinal cord injury and associated complications of methylprednisolone therapy: a national population-based study in South Korea. Spinal Cord. 2020;58:232-7.

2. Jeon I, Leigh J-H, Ro J-S, Ro YS, Lee SH, Shin H-I, et al. Trends in the incidence of work-related traumatic limb amputations in
South Korea from 2004 to 2013. Prosthet Orthot Int. 2019;43:409-17.

3. Korea Insurance Development Institute. 2018 analysis of automobile insurance subscription propensity. Wonju, Republic of Korea: Korea Insurance Development Institute; 2019.

4. Health Insurance Review \& Assessment Service. 2017 guide to the medical review and assessment. Seoul, Republic of Korea: Health Insurance Review \& Assessment Service; 2017.

5. Kim T-Y, Jang S, Park C-M, Lee A, Lee Y-K, Kim H-Y, et al. Trends of incidence, mortality, and future projection of spinal fractures in Korea using nationwide claims data. J Korean Med Sci. 2016;31:801-5. 